\section{Follow up of people recently discharged from psychiatric inpatient care}

Dear Editor - The period immediately following discharge from inpatient care is internationally recognised as a time of increased vulnerability to suicide. ' In the United Kingdom, the National Confidential Enquiry ${ }^{2}$ found high rates of suicide following discharge from psychiatric inpatient care. The rate was highest in the few days post discharge with $41 \%$ of suicides occurring before the first follow up appointment.

The report recommended follow up within 48 hours of discharge for high-risk patients and within seven days for all patients. In addition, the National Service Framework for Mental Health ${ }^{3}$ in Standard 7, Preventing Suicide, stated that "Care plans for those with severe mental illness include an urgent follow up within one week of discharge from hospital."

We considered whether any high-risk group of recently discharged inpatients should be specifically targeted. However, a study of patients who had committed suicide after discharge from hospital found only a third had severe mental illness and $40 \%$ were women. ${ }^{4}$ These people might not have been identified as at high risk of suicide.

Crawford ${ }^{5}$ confirmed that efforts to prevent suicide after an episode of inpatient care should not be focussed on specific high-risk groups but should target all patients recently discharged from hospital. We therefore decided to attempt early follow up of all patients recently discharged. Early follow up provides a key opportunity for suicide prevention.

This audit had one primary aim: to improve the rate of follow up assessment of patients within the first week after discharge from inpatient care.

Secondary aims were to improve the pre and post discharge practice with regard to the assessment of suicide risk, making appropriate interventions and documenting the details of these.

The authors developed a proforma to apply to case notes. This included some items from Preventing suicide: a toolkit for mental health services. ${ }^{6}$ Trust audit committee approval was granted. A retrospective audit was performed on a consecutive sample of 50 case notes of inpatients discharged to three community mental health teams (CMHTs) over four month period. The catchment area of the ward was urban, socially mixed and ethnically diverse, with relatively high concentrations of social housing and refugees and asylum seekers.

The service had a highly developed crisis and home treatment team and an assertive outreach teams, so was able to provide alternatives to admission for many patients in relapse.

The findings of the audit were disseminated via the following means:

- A presentation to the business meeting of the three CMHTs who participated in the audit

- Written and verbal reports to the trust audit committee

- Presentation to the trust Serious Incident Peer Group Meeting. For a full description of the function of the meetings, see Rose ${ }^{7}$

- Written report to the Adult Directorate Strategy Group.

An agreement was reached by members of the three teams to work toward the standard of seeing all inpatients with mental disorders within a week of inpatient discharge. A reaudit was performed using a similar methodology a year later on the records of 52 consecutively discharged patients. The standard was later adopted across the adult directorate and incorporated into the operational policy.

The inclusion of missing data may have slightly biased results against the second audit as there were three casenotes with incomplete information in the second audit compared to one only in the first one.

Both samples contained similar proportions of male and female patients, similar mean ages ( 39 and 36 years respectively), the same mean lengths of admission (52 days) and a similar range of diagnoses. Although the second sample contained more patients with schizophrenia (46\%) than the first (40\%), this difference was not significant at the $5 \%$ level. Similar proportions had deliberately harmed themselves in the three months prior to admission and initiated their own discharges.

In both audit samples, there were high rates $(64 \%$ first audit, $67 \%$ second audit) of pre-discharge meetings being held with inpatient and community staff. Good practice was also achieved in the vast majority of patients who were prescribed a medication supply for two weeks or less (83\% first audit, $96 \%$ second). Lower rates of compliarice with standards were seen in: the filing of the care plan in the case notes $(52 \%$ and $44 \%)$; the attendance rates of the community key-workers at the pre discharge meetings (which deteriorated significantly from $70 \%$ to $42 \%, \chi^{2}=9.6, \mathrm{df}=1$, $p=0.01$ ) and the rate of enquiry about suicide in the pre discharge meetings ( $44 \%$ first audit, $38 \%$ second). These areas had not improved when the second audit was undertaken. The care plans seldom gave advice to GPs about prescribing quantities ( $5 \%$ in first audit, $6 \%$ in second audit), compliance issues or problems with engagement.

There was one suicide during the initial audit period (this occurred two days after discharge) and no suicides occurred in the second audit phase. There were no records of non-fatal deliberate self-harm during either period. Significant improvements were made with regard to the early follow up of patients from the first to the second audits. This applied to both time frames, within two days (from $8 \%$ to $25 \%, \chi^{2}=4.1$ Yates' correction, $d f=1, p=0.05$ ) and seven days (from $48 \%$ to $73 \%, \chi^{2}=6.7, d f=1, p=0.01$ ). When follow up did not happen within seven days, the most common reason in both audits was that no appointment was made.

There was a reduction of the numbers of patients who were not given follow up appointments from $34 \%$ to $19 \%$ but this did not reach significance. Failure to follow up was much less likely to be due to the patient's non-attendance than failure of the service to give an appointment and this reduced from $14 \%$ to $8 \%$.

The whole range of professionals was involved in the follow up of patients. Community psychiatric nurses saw the largest numbers of patients in both samples $(62 \%$ and $39 \%$ respectively) and increased the number of patients they followed up. Doctors, the crisis team and to a lesser extent, social workers, all contributed to improved rates of early follow up. Interventions occurred only in a minority of the post discharge follow up reviews, and the rate at which interventions were 
made decreased from $38 \%$ in the first audit to $29 \%$ in the second. Interventions made included: assessment of suicide risk, involvement of other professionals, medication change, closer monitoring and detection of non-compliance.

The main strengths of this audit are the use of robust and well-respected standards and a standardised audit tool. It was further strengthened by the use of many sources of information for example, case notes, computerised information system, medicine charts and care plans. It used a variety of strategies to disseminate the findings and had a strong multidisciplinary ownership.

The main limitation is the retrospective nature of the data collection and the limited validity of the case notes at reflecting actual clinical practice with the inevitability that some data were missing. The sample size was small, limited to 50 patients in the first sample and 52 in the re-audit. This was because the audit was performed on one inpatient ward which was admitted to by three community mental health teams. To achieve a larger sample would have required recruitment of patients over a much longer period of time than the four month periods. Despite the small numbers, they were sufficient to show statistically significant changes (at the $1 \%$ level) to the primary aim: the follow up of patients within a week of discharge.

When attempting to generalise the results to other services, it is necessary to consider certain aspects of the service. Most significantly, there have been well developed community mental health teams and crisis teams operating for many years enabling patients to be managed at home wherever possible. There has also been a strong focus on continuity of care with the same consultants responsible for patients whether in the community or in hospital. Whether the findings of this audit would be so easily reproduced in services with different configurations is not clear.

This audit succeeded in the achievement of its primary aim, to improve the follow up of all patients recently discharged. This was achieved by the teams' acceptance of the standard and strong multi disciplinary working. Improvement in followup rates involved increased use of medical appointments and the crisis team. Doctors' posts in this trust span both inpatient and community services and they are therefore able to provide continuity of care. The compliance rate of follow up within one week of discharge did not, however, reach $100 \%$. A possible reason for this may have been the pre discharge practice, in particular the frequent failure of community key workers to attend the pre discharge meetings.

The other clear finding from this audit is that patients generally attended appointments that were given to them. Failure to meet the standard that all patients should be followed up within one week of their discharge from hospital was primarily due to deficiencies in the staff's organisational practice and therefore should be readily amenable to change.

These secondary aims have strong face validity in preventing suicide, although their actual effectiveness and cost effectiveness is open to question. Geddes ${ }^{8}$ argues that given the rarity of suicide and the limited sensitivity and specificity of current risk assessments, even if the sensitivity and specificity were assumed to be $80 \%$, for 20,000 patients discharged, 4,024 patients would be considered to be high risk, 3,992 of whom would be false positives. Nevertheless, there is good evidence that the period post discharge is when psychiatric inpatients are at their most vulnerable, and arguably when contact with the psychiatric services is most likely to be needed.

The main implication for service development is that clinicians and managers need to follow up patients assertively in the first week after discharge from hospital, which is known to be a vulnerable period. Improvements in the organisation of care can be achieved by close multidisciplinary working linked with increased use of crisis teams. Crisis teams have already shown to be successful at enabling people with acute mental health problems to remain at home. ${ }^{9}$ They also have a clear role in facilitating discharge which needs further evaluation.

Future audit needs to be broadened to ensure that an appropriate risk assessment has been made, and enquiry has been made with respect to specific risk factors. Follow up of psychiatric inpatients would be improved by specific guidelines directing clinicians to assess risk in every patient, modify the care plan accordingly and to document these processes. Early follow up may also be a useful means of preventing violence in recently discharged patients, a period of increased risk. ${ }^{10}$

Since the completion of this audit, the National Confidential Enquiry has reported again in December 2006. It confirms that the early post discharge period remains one of increased suicide risk and continues to recommend follow up within a week of leaving hospital. Furthermore, it highlights other factors indicating high risk such as short admission durations, readmissions and self-discharge. These vulnerable groups of people should receive highest priority for community mental health resources on leaving hospital which could be evaluated by similar audits.

Maria G. Turri,

Senior House Officer in Psychiatry Oxfordshire Mental Healthcare NHS Trust, England maria.turri@oxmhc-tr.nhs.uk

Preeti Chhabra

Senior House Officer in Psychiatry Broadmoor Hospital, Crowthorne, Berkshire, RG45 7EG, England

Robert Chaplin,

Consultant Psychiatrist Oxfordshire Mental Healthcare NHS Trust, UK Research Fellow, Royal College of Psychiatrists' Research Unit, London, SW1H OHW, England

References

1. Pompili M, Mancinelli t, Ruberto A, Kotzalidis GD, Girardi P, Tatarelli R. Where schizophrenic patients commit suicide: a review of suicide among inpatients and former inpatients. International Journal of Psychiatry in Medicine 2005; 35: 171-190

2. Safety first: Five year report of the Confidential Enquiry into Suicide and Homicide by People with Mental lilness. London: Department of Health 2001

3. Department of Health. National Service Framework for Mental Health: Modern standards and service models. London: Department of Health 1999

4. Goldacre M, Seagroatt V, Hawton, K. Suicide after discharge from psychiatric inpatient care. Lancet 1993; 342: 283-286

5. Crawford M.J. Suicide following discharge from in-patient psychiatric care. Adv Psych Treat 2004; 10: 434-438

6. National Institute for Mental Health in England (NIHME). Preventing suicide: A toolkit for mental health services 2003. Website accessed 07.03.2006 http://kc.nimhe.org.uk/upload/SuicidePreventionToolkitweb.pdf

7. Rose N. Six years' experience in Oxford: Review of serious incidents. Psych Bull 7. Rose N. Six years

8. Geddes J. Suicide and homicide by people with mental iliness. BMJ 1999; 318 1225-1226

9. Johnson S, Nolan F, Pilling S, et al. Randomised controlled trial of acute mental health care by a crisis resolution team: the north Islington crisis study. BMJ 2005; 331 : 599-602

10. Steadman HJ, Mulvey EP, Monahan J, et al. Violence by people discharged from acute psychiatric inpatient facilities and by others in the same neighborhoods. Arch Gen Psychiat 1998; 55: 393-401 